\title{
Transport in cleaved-edge overgrowth wires
}

\author{
R. de Picciotto ${ }^{\mathrm{a}, *}$, H.L. Stormer ${ }^{\mathrm{a}, \mathrm{b}, \mathrm{c}}$, A. Yacoby ${ }^{\mathrm{a}, \mathrm{d}}$, L.N. Pfeiffer ${ }^{\mathrm{a}}$, K.W. Baldwin ${ }^{\mathrm{a}}$, K.W. West ${ }^{\mathrm{a}}$ \\ ${ }^{a}$ Bell-Labs, Lucent Technologies, 600 Mountain Ave., RM. IC-329, Murray Hill, NJ 07974, USA \\ ${ }^{\mathrm{b}}$ Department of Physics, Columbia University, New York, NY 10003, USA \\ c Department of Applied Physics, Columbia University, New York, NY 10003, USA \\ ${ }^{\mathrm{d}}$ Braun Center for Submicron Research, Dept. of Condensed Matter Physics, Weizmann Institute of Science, Rehovot 76100 , Israel
}

\begin{abstract}
We report the results of 3 terminal measurements on a one-dimensional (1D) wire. By utilizing cleaved-edge overgrowth, we were able to vary the coupling between a 1D conductor and an adjacent two-dimensional electron gas (2DEG) strip attached to the central part of the wire. We study the 1D-2DEG coupling by controlling the width of the 2DEG strip. Ballistic transport along the wire persists for interaction regions as long as $6 \mu \mathrm{m}$. Thus, with our shortest interaction region (narrowest strip) of $2 \mu \mathrm{m}$, the wire remains almost undisturbed by the presence of the nearby 2DEG allowing a true 3 terminal measurement on a clean wire. Our observations also explain the origin of the non-universal conductance quantization of such 1D wires. (c) 2001 Elsevier Science B.V. All rights reserved.
\end{abstract}

Keywords: One-dimensional wires; Ballistic transport; Quantum conductance

An ideal Ohmic contact to a conductor should fill all outgoing states up to an electro-chemical potential $\mu$ and absorb all incoming particles. In 1D this can be achieved by using an adiabatic funnel, which slowly reduces the cross section of a two- or three-dimensional conductor, forming the 1D wire at its end. The adiabatic funnel avoids reflections at the wire entrance and thus ensures the filling of all the outgoing states [1]. Contacted with such funnels at both ends, a clean wire should exhibit a conductance that is quantized in multiples of a universal value $g_{0}=2 e^{2} / h[2-12]$. The conductance increases in a series of steps as subsequent $1 \mathrm{D}$ electronic sub-bands

\footnotetext{
${ }^{*}$ Corresponding author. Tel./fax: +1-908-382-3981.

E-mail address: rd25@lucent.com (R. de Picciotto).
}

are filled, with plateau values equal to the universal conductance multiplied by the number of occupied wire modes.

Nearly adiabatic funnels can be fabricated in semiconductor structures by using two lithographically defined gates to progressively narrow a two-dimensional electron gas (2DEG) in order to form a wire [13]. When the distance between the funnels is shrunk to a point, as in quantum point contacts, essentially quantized conductance values can be observed $[14,15]$. However, 1D wires of a finite length that are fabricated by lithography suffer from small 1D sub-band-energy separation leading to possible mode mixing. Moreover, random width fluctuations can create discrete quantum point contacts along the wire that may dominate the conductance. 


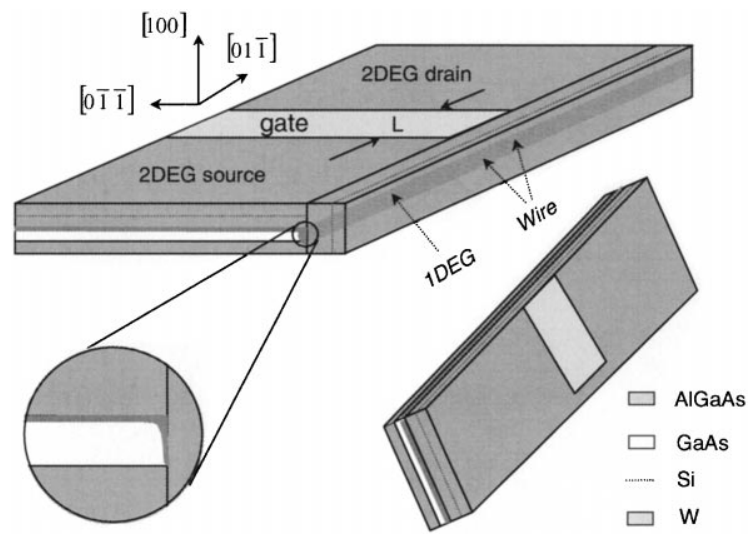

Fig. 1. The geometry of a two-terminal cleaved-edge overgrowth device (see text).

Cleaved-edge overgrowth (CEO) in Molecular Beam Epitaxy (MBE) [16] has proven to create 1D wires that are very homogeneous and have strong confinement. They show well-quantized conductance plateaus [17-19] but the step height can be as much as $25 \%$ less than the expected universal value. It has been suggested that the observed non-universality is associated either with electron scattering between the 1D wire and its 2D contacts [17] or with disorder combined with e-e interactions inside the wire $[17,19]$. However, since this behavior is observed in wires as short as $1-2 \mu \mathrm{m}$ - much shorter than the back scattering length in such high-quality CEO wires [17] - 1D back scattering alone cannot account for the deviation from universal conductance. Indeed e-e interactions are expected to strongly affect the two terminal conductance of non-ideal 1D electronic systems, be it due to non-idealities inside the wire or at the contacts. However, the inclusion of e-e interactions is not expected to alter the universal quantized conductance in clean wires with ideal contacts [8$12]$. We thus concentrate here on the properties of the electrical contacts employed in CEO.

Wires fabricated by CEO are contacted via a $2 \mathrm{DEG}$, as illustrated in Fig. 1. A high-quality 2DEG is created by MBE growth of a unilaterally doped GaAs quantum well (QW) onto a [0 01 1] GaAs substrate. In our devices, this $2 \mathrm{DEG}$ has a carrier density $n_{\mathrm{s}} \approx$ $2.5 \times 10^{11} \mathrm{~cm}^{-2}$, and mobility $\mu \approx 4 \times 10^{6} \mathrm{~cm}^{2} / \mathrm{Vs}$. Loaded inside the MBE chamber this wafer is cleaved

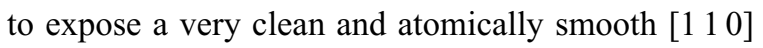

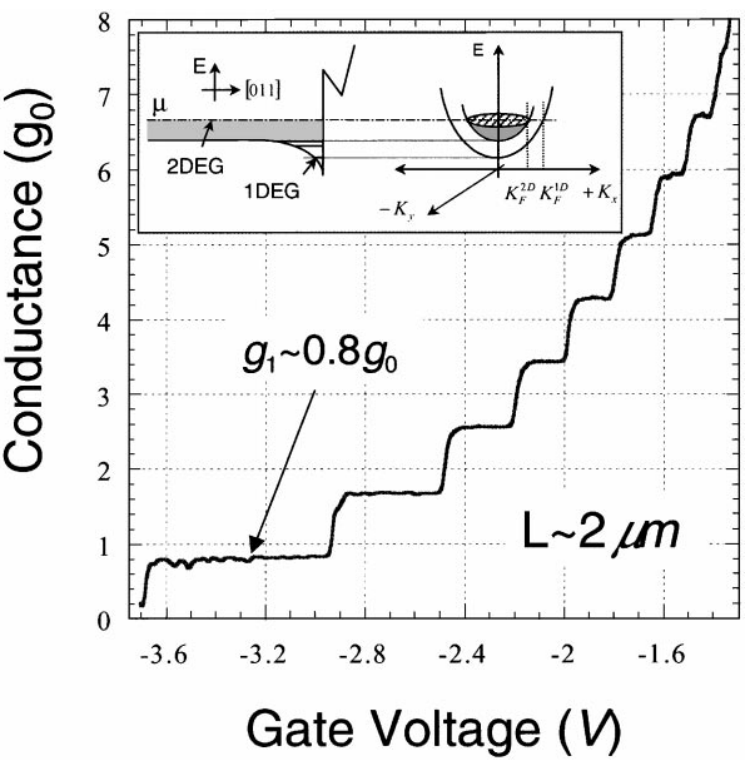

Fig. 2. Two-terminal conductance of a CEO wire. Solid line: two-terminal conductance, $g_{1}$, of a $2 \mu \mathrm{m}$ long wire, plotted against the voltage applied to the gate. Inset: Left-hand side: the profile of the conduction band in the GaAs quantum well near the cleaved edge and away from the gates. The 2DEG and the 1DEG modes are shown. Right-hand side: dispersion relation for electrons in the 2DEG and 1D wire. The Fermi wave vectors of the two systems are indicated.

surface, which is immediately overgrown with a second modulation-doped epitaxial layer sequence. The additional remote $\mathrm{Si}$ dopants that are introduced in this latter step lead to a higher electron density near the cleaved edge of the QW. As in conventional modulation doped samples, a strong built in electric field confines this excess charge to the cleaved edge interface - leading to the formation of a high-quality $1 \mathrm{D}$ channel, containing $\sim 10$ electronic modes that are bound to the cleaved edge (see inset of Fig. 2). We refer to this channel as the "1DEG". It exists all along the cleaved edge of the QW in which the 2DEG resides. A gate electrode is used to locally deplete the 2DEG, leaving only the $1 \mathrm{DEG}$ along the edge to connect between the 2DEGs on both sides, which are used as two-terminal contacts. We refer to this isolated $1 \mathrm{DEG}$ in front of the gate as the "wire". The length, $L$, of the wire is defined by the width of the gate. Increasing the gate voltage beyond depletion of the 2DEG provides a convenient tool to control the number of occupied 1D-wire modes while the source drain con- 
ductance is being measured. A typical result of such a two terminal (2T) conductance measurement, $g_{1}$, is shown in Fig. 2. A standard lock-in technique with an excitation current smaller than $1 \mathrm{nA}$ at a temperature, $\theta \approx 300 \mathrm{mK}$ was used. We observe clear conductance plateaus as a function of gate voltage with a plateau height of $\sim 0.8 g_{0}$ - smaller than the expected value $g_{0}$. This result is essentially the same as the one obtained in Ref. [17].

In this contact configuration, the 1DEG is coupled to the 2DEG on either side of the wire for practically an infinite length (namely the crystal size, which is a few $\mathrm{mm}$ ) - seemingly insuring good 1D-2D coupling. However, an electron that is transferred from the 2DEG into the 1DEG far away from the entrance to the wire might be reflected within the 1DEG or scattered back into the 2DEG. Therefore, the competition between the 2D-1D scattering rate and the $1 \mathrm{D}$ back scattering rate establishes an effective contact length and determines the efficiency with which the outgoing 1D states are filled by the source.

A traditional 2T measurement cannot determine the contact contribution to the overall conductance. In order to study the properties of the contacts used in CEO wires, we split the top gate in two (gates A and B in the inset of Fig. 3), creating between the gates a section of controllable width, $W$, where a 2DEG strip taps into the 1DEG. This allows us to determine the length scale over which the 2DEG reservoir couples efficiently to the $1 D E G$ and a good contact is formed. Transport data for such a device is shown in Fig. 3. We use one gate to allow only a single mode in wire $\mathrm{B}$ while the voltage applied to gate $\mathrm{A}$ is scanned. The overall conductance, $g_{2}$, of the two wires plus a (floating) $W \sim 2 \mu \mathrm{m}$ long tap in series is shown by the solid line in this figure. When both wires support a single mode (shaded region), we find $g_{2} \approx 0.75 g_{0} \approx$ $0.94 g_{1}$ - clearly demonstrating ballistic $1 \mathrm{D}$ transport through the $2 \mu \mathrm{m}$ long tap. For a $W \sim 10 \mu \mathrm{m}$ tap we find a much smaller $g_{2},\left(g_{2} \approx 0.4 g_{0} \approx \frac{1}{2} g_{1}-\right.$ not shown). This result indicates complete thermalization in the tap [2-4]. Such thermalization leads to a classical (Ohmic) combination of the resistances of the two wires in series. For an intermediate tap length of $W \sim 6 \mu \mathrm{m}$ we find $g_{2} \approx 0.6 g_{0} \approx 0.75 g_{1}$ (not shown). This value falls half way between the "Ohmic tap" result, $\frac{1}{2} g_{1}$, and the "no tap" value, $g_{1}$. This is the central result of the present study. By varying the tap length

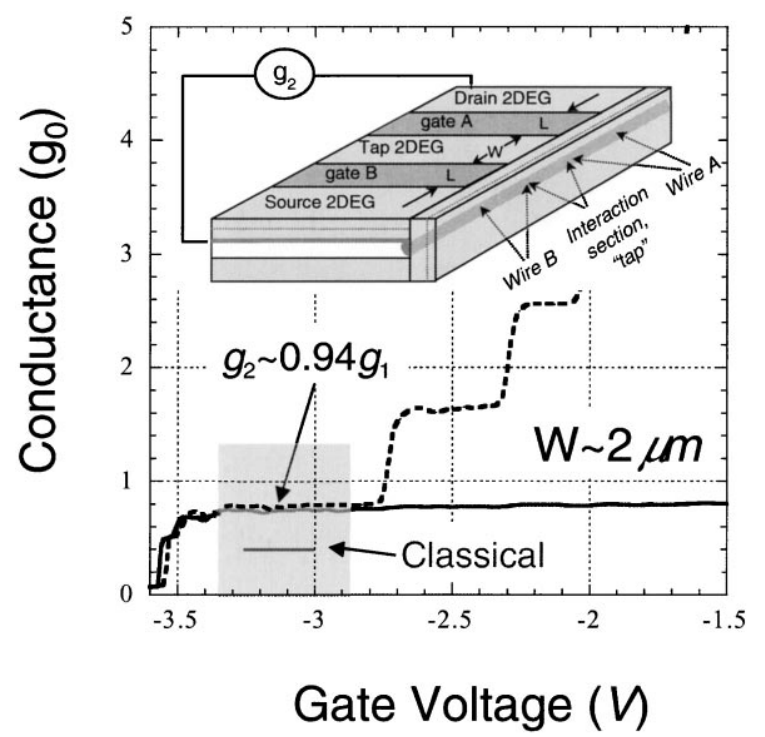

Fig. 3. Connecting wires in series. Solid line: conductance, $g_{2}$, of two wires in series with a $2 \mu \mathrm{m}$ long floating tap plotted versus the voltage applied to gate A while wire B sustains only one mode. The shaded region corresponds to a single mode in both wires A and B. Dashed line: two-terminal conductance, $g_{1}$, of the $2 \mu \mathrm{m}$ long wire A, plotted against the voltage applied to gate A. Similar results are obtained for wire B (not shown). These latter measurements employ one gate (A or B). Inset: The measurement scheme is shown along with the geometry of a three-terminal CEO device (see text).

we can follow the changeover from a classical addition rule (invasive tap) to a "universal" value $\left(0.8 g_{0}\right.$ in the present case) for a non-invasive tap [4].

The smaller value of $g_{2}$ as compared to $g_{1}$ indicates additional $1 \mathrm{DEG}$ back scattering induced by the presence of the tap 2DEG. Since this 2DEG is floating, an electron that scatters from the $1 \mathrm{DEG}$ into the tap 2DEG is replaced on average by an electron that scatters in the reverse direction. This latter electron, however, has an equal probability to scatter into either direction; $+k_{\mathrm{F}}^{1 \mathrm{D}}$ or $-k_{\mathrm{F}}^{1 \mathrm{D}}-$ resulting in $1 \mathrm{D}$ momentum loss. Note that 2DEG-1D e-e scattering that involves momentum exchange without charge transfer cannot occur because $k_{\mathrm{F}}^{2 \mathrm{D}}<k_{\mathrm{F}}^{1 \mathrm{D}}$ (see inset of Fig. 2 ). For the same reason, the $2 \mathrm{DEG}$ screening of any disorder potential at the wave vectors relevant for $1 \mathrm{D}$ back scattering $\left(2 k_{\mathrm{F}}^{1 \mathrm{D}}\right)$ is very inefficient; hence, the back scattering length in a wire, $l_{\mathrm{B}}^{\text {wire }}$, is expected to be very similar to the one in the $1 \mathrm{DEG}, l_{\mathrm{B}}^{1 \mathrm{DEG}}$. Therefore, for the geometry of this experiment the additional 
Table 1

Three-terminal measurements on a 1D wire and comparison with predictions of a model (see text). All measurements are performed with a single mode in both wires. The configurations of the various measurements are illustrated. The applied voltages are $V_{\mathrm{s}}=\mu_{\mathrm{s}}-\mu_{\mathrm{d}}$ and $V_{\mathrm{t}}=\mu_{\mathrm{t}}-\mu_{\mathrm{d}}$ with $\mu_{\mathrm{s}}, \mu_{\mathrm{d}}$ and $\mu_{\mathrm{t}}$ the electro-chemical potentials of the 2DEG in the source, drain and tap

\begin{tabular}{lll}
\hline Measurement scheme & Measured $( \pm 3 \%)$ & Model $(\alpha=0.8, T=0.14)$
\end{tabular}

a.

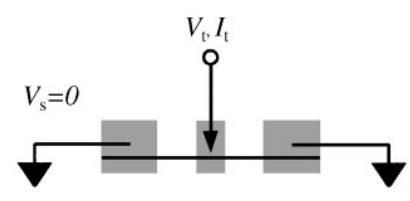

$$
\left(g_{0}\right)^{-1} I_{\mathrm{t}} /\left.V_{\mathrm{t}}\right|_{V_{\mathrm{s}}=0}=0.22
$$

b.

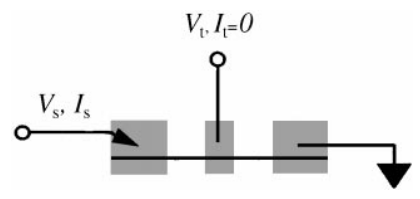

c.

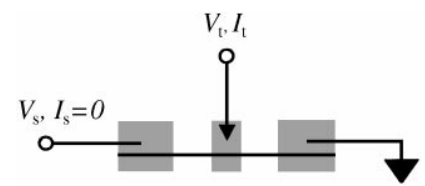

$\left(g_{0}\right)^{-1} I_{\mathrm{S}} /\left.V_{\mathrm{s}}\right|_{I_{\mathrm{t}}=0}=0.75$

$\left(g_{0}\right)^{-1} I_{\mathrm{s}} /\left.V_{\mathrm{t}}\right|_{I_{\mathrm{t}}=0}=1.51$

$\alpha\left(1-\frac{1}{2} T\right)=0.74$

$2 \alpha\left(1-\frac{1}{2} T\right)=1.49$

$$
\begin{array}{ll}
\left(g_{0}\right)^{-1} I_{\mathrm{t}} /\left.V_{\mathrm{t}}\right|_{I_{\mathrm{s}}=0}=0.21 & \alpha\left(2 T-T^{2}\right)=0.21 \\
\left(g_{0}\right)^{-1} I_{\mathrm{t}} /\left.V_{\mathrm{s}}\right|_{I_{\mathrm{s}}=0}=1.49 & 2 \alpha\left(1-\frac{1}{2} T\right)=1.49
\end{array}
$$

back scattering induced by the tap is predominantly due to charge transfer between the two subsystems and hence measures the amount of 1D-2D scattering that takes place for a given tap length. Our measurements thus allow us to deduce a 2D-1D mean free path of $l_{2 \mathrm{D} \leftrightarrow 1 \mathrm{D}} \approx 6 \mu \mathrm{m}$. This scattering length establishes the quality of the contacts and hence determines the 2T-conductance $g_{1}$.

The invasiveness of the tap can be also determined by measuring of the 2T-tap conductance; $g_{\mathrm{t}}=I_{\mathrm{t}} / V_{\mathrm{t}}$. This is achieved by driving the current from the tap into both source and drain. For $W \sim 10 \mu \mathrm{m}$ we find $g_{\mathrm{t}} \approx 2 g_{1}$, as expected for an Ohmic combination of the two wires in parallel. For $W \sim 2 \mu \mathrm{m}$, the value of $g_{\mathrm{t}}$ is much smaller; $g_{\mathrm{t}} \approx 0.22 g_{0}$, indicating a small 2DEG-1D transmission for such a short tap.

Previous work on CEO wires [17] derived an expression for the expected 2T-conductance; $g_{1}=g_{0}\left(1+2 l_{2 \mathrm{D} \leftrightarrow 1 \mathrm{D}} / l_{\mathrm{B}}^{1 \mathrm{DEG}}\right)^{-1 / 2}$, in terms of the two quantities $-l_{\mathrm{B}}^{1 \mathrm{DEG}}$ and $l_{2 \mathrm{D} \leftrightarrow 1 \mathrm{D}}$. Here we measure $l_{2 \mathrm{D} \leftrightarrow 1 \mathrm{D}}$ directly - not possible via traditional 2T-measurements. Using $l_{2 \mathrm{D} \leftrightarrow 1 \mathrm{D}} \approx 6 \mu \mathrm{m}$ and the $1 \mathrm{D}$ back scattering length $l_{\mathrm{B}}^{1 \mathrm{DEG}} \approx l_{\mathrm{B}}^{\text {wire }} \approx 20 \mu \mathrm{m}$ [17] in the above expression yields $g_{1} \approx 0.79 g_{0}$ for the calculated $2 \mathrm{~T}$-conductance - in excellent agreement with the measured value; $g_{1} \approx 0.8 g_{0}$. The origin of the non-universal conductance in CEO wires is thus mainly 2D-1D scattering at the regions of the contacts. The contribution of such non-ideal contact to the overall conductance is expected to be affected by e-e interaction and thus to depend on temperature and applied voltage. Nevertheless, the linear response data can still be described in terms of the simple model above, namely in terms of these two scattering rates.

Our device allows us to perform three-terminal (3T) measurements on a wire by using the tap as 
a third terminal. Such measurements have not been possible in 1D wires thus far. The results of such measurements, with a single mode in both wires, are presented in Table 1. We drive a current from source to drain and measure the tap voltage, $V_{\mathrm{t}}$. The 3T-conductance $g_{\mathrm{sd}, \mathrm{t}}=I_{\mathrm{s}} / V_{\mathrm{t}}$ equals twice the 2T-conductance $g_{2}=I_{\mathrm{s}} / V_{\mathrm{s}}$, which is obvious from the symmetry of our device. With the current driven from the tap and sunk at the drain we use the source terminal as a voltage probe. Here we find a similar 3T-conductance $g_{\mathrm{td}, \mathrm{s}}=I_{\mathrm{t}} / V_{\mathrm{s}} \approx 2 g_{2}$ - as expected from Onsager relations [6]. This less intuitive result is a consequence of the tap current flowing into both drain and source. The source 2DEG reacts by increasing its electro-chemical potential to a value needed to send an equal current in the opposite direction to satisfy the zero source current condition.

In order to interpret our 3T linear response data, we adopt the Landauer scattering approach [3-6]. We focus here on a single 1D mode and neglect mode mixing. Non-ideal 2D-1D coupling at the source and drain is modeled with a reduced contact emissivity, $\alpha$. The finite length of the tap, $W$, leads to a yet smaller tap emissivity, $\alpha T(W)$, where $T(W)$ is the transmission probability from the tap $2 \mathrm{DEG}$ into either direction in the first 1 DEG mode. Assuming symmetric coupling to both sides, the scattering matrix of the junction depends on these two parameters alone, leading to

$$
\begin{aligned}
& I_{\mathrm{s}}=g_{0} \alpha\left(V_{\mathrm{s}}-T V_{\mathrm{t}}\right), \\
& I_{\mathrm{t}}=g_{0} \alpha T\left(2 V_{\mathrm{t}}-V_{\mathrm{s}}\right) .
\end{aligned}
$$

This model is equivalent to the approach taken in Ref. [17] when modified to accommodate the additional tap reservoir. The values of $\alpha$ and $T$ are deduced from the measurements of $g_{1}$ and $g_{\mathrm{t}}$ as described above. We find $\alpha=g_{1} / g_{0} \approx 0.8$ and $T=g_{\mathrm{t}} / 2 g_{1} \approx 0.14$ for $W \sim$ $2 \mu \mathrm{m}$. Without additional adjustable parameters, we find excellent agreement between our 3T-results and this model, as illustrated in Table 1.

In conclusion, we were able to study the coupling between a $2 \mathrm{DEG}$ and a $1 \mathrm{D}$ wire. We find a scat- tering mean free path of $\sim 6 \mu \mathrm{m}$. Our results show that the origin of the non-universal conductance previously observed in such wires is electron scattering across the 2DEG-1DEG interface at the contacts. Past the well-defined contact geometry in CEO, traditional evaporated metal contacts to one-dimensional molecules such as carbon nanotubes, which are conjectured to inject and remove electrons all-along the contact length, may be subject to similar mechanisms.

\section{References}

[1] L.I. Glazman, G.B. Lesovik, D.E. Khmel'nitskii, R.I. Shekhter, JEPT Lett. 48 (1988) 238.

[2] Y. Imry, Introduction to Mesoscopic Physics, Oxford University Press, Oxford, 1997.

[3] R. Landauer, IBM J. Res. Dev. 32 (1988) 306.

[4] M. Buttiker, Phys. Rev. Lett. 33 (1986) 3020.

[5] M. Buttiker, Phys. Rev. Lett. 57 (1986) 1761.

[6] M. Buttiker, IBM J. Res. Dev. 32 (1988) 317.

[7] C.W.J. Beenakker, H. von Houten, in: H. Ehrenreich, D. Turnbull (Eds.), Solid State Physics, Semiconductor Heterostructures and Nanostructures, Academic Press, New York, 1991.

[8] I. Safi, H.J. Schulz, Phys. Rev. B 52 (1995) R17 040.

[9] V.V. Ponomarenko, Phys. Rev. B 52 (1995) R8666.

[10] D.L. Maslov, Phys. Rev. B 52 (1995) R14 368.

[11] Y. Oreg, A.M. Finkel'stein, Phys. Rev. B 54 (1996) 14265.

[12] A.Y. Alekseev, V.V. Cheianov, Phys. Rev. B 57 (1998) 12.

[13] S. Tarucha, T. Honda, T. Saku, Solid State Commun. 94 (1995) 413.

[14] B.J. van Wees, H. van Houten, C.W.J. Beenaker, J.G. Williamson, L.P. Kouwenhoven, D. van der Marel, C.T. Foxon, Phys. Rev. Lett. 60 (1988) 848.

[15] D.A. Wharam, T.J. Thornton, R. Newbury, M. Pepper, H. Ahmed, J.E.H. Frost, D.G. Hasko, D.C. Peacock, D.A. Ritchie, C.A.C. Jones, J. Phys. CI 21 (1988) L209.

[16] L.N. Pfeiffer, A. Yacoby, H.L. Stormer, K.L. Baldwin, J. Hasen, A Pinczuk, W. Wegscheider, K.W. West, Microelectron. J. 28 (1997) 817.

[17] A. Yacoby, H.L. Stormer, N.S. Wingreen, L.N. Pfeiffer, K.W. Baldwin, K.W. West, Phys. Rev. Lett. 77 (1996) 4612.

[18] A. Yacoby, H.L. Stormer, K.W. Baldwin, L.N. Pfeiffer, K.W. West, Solid State Commun. 101 (1997) 77.

[19] M. Rother, W. Wegscheider, M. Bichler, G. Abstreiter, in: D. Gershoni (Ed.), Proceedings of ICPS24, World Scientific, Singapore, 1998. 